\title{
On the existence of a Generalized Langevin model representation for second-moment closures
}

\author{
H. A. Wouters, ${ }^{\text {a) }}$ T. W. J. Peeters, and D. Roekaerts ${ }^{\text {b) }}$ \\ Section Heat Transfer, Department of Applied Physics, Delft University of Technology, \\ Lorentzweg 1, 2628 CJ, Delft, the Netherlands
}

(Received 14 February 1996; accepted 27 March 1996)

\begin{abstract}
The Generalized Langevin model representations of two second-moment closure models for the rapid pressure-strain term, proposed by Fu and Launder and by Jones and Musonge, are obtained. This representation makes it possible to use these models in PDF calculations of turbulent flows. The implications of three realizability constraints for the relationship between Langevin models and these second-moment closures are discussed. A Generalized Langevin model representation exists only if the rapid pressure-strain model satisfies realizability at the 2D turbulence limit. (C) 1996 American Institute of Physics. [S1070-6631(96)01807-7]
\end{abstract}

Langevin models provide a general and computationally attractive class of Lagrangian turbulence models to calculate the probability density function of velocities. ${ }^{1}$ The relationship between a generalized form of the Langevin model and conventional Eulerian second-moment closures was shown by Haworth and Pope ${ }^{2}$ and was exploited further by Pope. ${ }^{3}$ In this Letter this relationship is looked upon in detail for the rapid pressure-strain models of Fu and Launder ${ }^{4}$ (FL) and of Jones and Musonge ${ }^{5}(\mathrm{JM})$. Both models will be written in a general representation and the equivalent Langevin models will be presented. The FL model was developed from a general expression for the pressure-strain correlation which was reduced to a two parameter model by application of three realizability constraints. The JM model was developed specifically for variable density flows and may be useful for reacting flow simulations. This model satisfies the proper symmetry conditions but does not explicitly satisfy realizability. We want to make some remarks about the implications of the realizability constraints for the relationship between second-moment closures and Langevin models. This work can be seen as an additional remark to the work of Pope and the outline of this Letter will follow Pope $\mathrm{P}^{3}$ closely. It shows that non-linear second-moment closure models can be used in PDF calculations of turbulent flows.

$\mathrm{Fu}$ and Launder model the rapid-pressure strain term $\phi_{i j 2}$ as a function of the mean velocity gradients and anisotropy tensor by

$$
\phi_{i j 2}=2 k\left(a_{l i k j}+a_{l j k i}\right) \frac{\partial \bar{U}_{k}}{\partial x_{l}},
$$

in which $\bar{U}$ denotes the mean velocity, $k$ the turbulent kinetic energy, and the fourth-order tensor $a_{l i k j}$ is a function of the anisotropy tensor only. The Reynolds stress tensor is denoted by ${\overline{u_{i}}}_{j}$. A general expression for $a_{l i k j}$, which contained up to cubic terms in the anisotropy tensor, was reduced to a two parameter model by application of three kinematic constraints which require that the model is redistributive only $\left(a_{l i k i}=0\right)$, that it satisfies normalization $\left(a_{l i k k}=\overline{u_{i} u_{l}} / k\right)$ and that it is valid in the limit of two-dimensional turbulence $\left(\phi_{\alpha \alpha 2}=0\right.$ for $\left.\bar{u}_{\alpha} u_{\alpha} \rightarrow 0\right)$. The two model parameters are called $c_{2}$ and $c_{2}^{\prime}$ of which $c_{2}$ multiplies a purely rotational term and $c_{2}^{\prime}$ is important in near wall flows. The standard values $c_{2}=0.6$ and $c_{2}^{\prime}=0$ give good results for free shear flows but improved predictions were obtained taking $c_{2}=0.55$ and $c_{2}^{\prime}=0.6^{4}$

For convenience the model is represented on a basis of tensors polynomials $T_{i j}^{(n)}$ (see Table I) which were defined by Pope. ${ }^{3}$ Compared to the original basis $T_{i j}^{(3)}$ was modified so that its trace is zero in variable density flows and to represent terms in $c_{2}$ and $c_{2}^{\prime}$ tensors $T_{i j}^{(9)}$ and $T_{i j}^{(10)}$ were added, respectively. For FL the coefficients $A^{(n)}$ are given in Table II.

The coefficients $A^{(n)}$ are related to the coefficients of the Generalized Langevin model ${ }^{2}$ (GLM) by a set of linear algebraic equations. To represent terms in $T_{i j}^{(9)}$ and $T_{i j}^{(10)}$ the GLM was extended with quadratic terms in the anisotropy tensor $b_{i j}$ with coefficients $\xi$. This, more general, version of the GLM reads as

$$
d U_{i}=-\frac{1}{\rho} \frac{\partial \bar{p}}{\partial x_{i}} d t+G_{i j}\left(U_{j}-\bar{U}_{j}\right) d t+\left(C_{0} \epsilon\right)^{1 / 2} d W_{i},
$$

in which $\partial \bar{p} / \partial x$ is the mean pressure gradient, $U$ is the instantaneous velocity, $C_{0}$ is a positive constant, $W$ is an isotropic Wiener process and

$$
G_{i j}=\frac{\epsilon}{k}\left(\alpha_{1} \delta_{i j}+\alpha_{2} b_{i j}+\alpha_{3} b_{i j}^{2}\right)+H_{i j k l} \frac{\partial \bar{U}_{k}}{\partial x_{l}},
$$

in which $\epsilon$ is the dissipation of turbulent kinetic energy and

$$
\begin{aligned}
H_{i j k l}= & \beta_{1} \delta_{i j} \delta_{k l}+\beta_{2} \delta_{i k} \delta_{j l}+\beta_{3} \delta_{i l} \delta_{j k}+\gamma_{1} \delta_{i j} b_{k l} \\
& +\gamma_{2} \delta_{i k} b_{j l}+\gamma_{3} \delta_{i l} b_{j k}+\gamma_{4} b_{i j} \delta_{k l}+\gamma_{5} b_{i k} \delta_{j l} \\
& +\gamma_{6} b_{i l} \delta_{j k}+\xi_{1} b_{i j} b_{k l}+\xi_{2} b_{i k} b_{j l}+\xi_{3} b_{i l} b_{j k} .
\end{aligned}
$$

The 15 coefficients $\alpha, \beta, \gamma$, and $\xi$ can be functions of scalar invariants of $b_{i j}$ and the strain tensor $S_{i j}$. Terms in $\beta$, $\gamma$, and $\xi$ multiplying $\delta_{i j}$ or $b_{i j}$ and can be chosen arbitrarily because these can be expressed in $\alpha_{1}$ or $\alpha_{2}$, respectively, or, the other way around, terms in $\alpha_{1}$ and $\alpha_{2}$ which contain scalar invariants of $S_{i j}$ can be expressed in $\beta, \gamma$, and $\xi$. Equating the modeled Reynolds stress equations, for both the conventional and the Lagrangian model, the modeled redistribution term $\phi_{i j}$ yields $^{2}$ 
TABLE I. Non-dimensional, symmetric tensors $T_{i j}^{(n)}$ which are functions of the anisotropy, strain, and rotation tensors $b_{i j}, S_{i j}$, and $W_{i j}$, respectively [see Pope (Ref. 3)].

$$
\begin{aligned}
& T_{i j}^{(1)}=b_{i j} \\
& T_{i j}^{(2)}=b_{i j}^{2}-\frac{1}{3} b_{l l}^{2} \delta_{i j} \\
& T_{i j}^{(3)}=S_{i j}-\frac{1}{3} S_{l l} \delta_{i j} \\
& T_{i j}^{(4)}=S_{i l} b_{l j}+S_{j l} b_{l i}-\frac{2}{3} S_{l m} b_{m l} \delta_{i j} \\
& T_{i j}^{(5)}=W_{i l} b_{l j}+W_{j l} b_{l i} \\
& T_{i j}^{(6)}=S_{i l} b_{l j}^{2}+S_{j l} b_{l i}^{2}-\frac{2}{3} S_{l m} b_{m l}^{2} \delta_{i j} \\
& T_{i j}^{(7)}=W_{i l} b_{l j}^{2}+W_{j l} b_{l i}^{2} \\
& T_{i j}^{(8)}=b_{i l} S_{l m} b_{m j}-\frac{1}{3} S_{l m} b_{m l}^{2} \delta_{i j} \\
& T_{i j}^{(9)}=b_{i k}^{2} W_{k l} b_{l j}+b_{j k}^{2} W_{k l} b_{l i} \\
& T_{i j}^{(10)}=b_{i k}^{2} S_{k l} b_{l j}+b_{j k}^{2} S_{k l} b_{l i}-\frac{2}{3} b_{l k}^{2} S_{k m} b_{m l} \delta_{i j}
\end{aligned}
$$

$$
\phi_{i j}=\left(\frac{2}{3}+C_{0}\right) \epsilon \delta_{i j}+G_{i l}{\overline{u_{l} u}}_{j}+G_{j l}{\overline{u_{l} u_{i}}}
$$

which includes both rapid and slow pressure-strain effects as well as effects of anisotropy of dissipation. Choosing $\beta_{1}, \gamma_{1}$, $\gamma_{4}$, and $\xi_{1}$, the eight remaining coefficients $\beta, \gamma$, and $\xi$ are related to the coefficients $A^{(3)}$ through $A^{(10)}$. This set of equations has a rank deficiency of one and obtains a one parameter solution if

$$
A^{*} \equiv \frac{3}{2} A^{(3)}-A^{(4)}+\frac{1}{3} A^{(6)}+\frac{1}{6} A^{(8)}-\frac{1}{9} A^{(10)}=0 .
$$

A GLM representation of a second-moment closure exists only if Eq. (6) is satisfied. Apart from the fact that this condition on the second-moment closure follows from algebraic derivations it has a physical background which will be shown below. Equation (6) is satisfied by FL for all $c_{2}$ and $c_{2}^{\prime}$. The corresponding GLM coefficients $\beta, \gamma$, and $\xi$ are given in Table III. Having solved for $\beta, \gamma$, and $\xi$ the coefficients $\alpha$ are determined by the relations to $A^{(1)}$ and $A^{(2)}$ and by the fact that the modeled redistribution term may not affect the level of turbulent kinetic energy.

Jones and Musonge model the pressure-strain correlation by

$$
\begin{aligned}
\phi_{i j 2}= & \frac{2}{3} c_{2}\left(D_{i j}-\frac{1}{3} P_{k k} \delta_{i j}\right)+c_{3}\left(D_{i j}-P_{i j}\right) \\
& +c_{4}\left(\frac{\partial \bar{U}_{i}}{\partial x_{j}}+\frac{\partial \bar{U}_{j}}{\partial x_{i}}-\frac{2}{3} \frac{\partial \bar{U}_{k}}{\partial x_{k}} \delta_{i j}\right)+2 c_{5} b_{i j} \frac{\partial \bar{U}_{k}}{\partial x_{k}}
\end{aligned}
$$

in which $D_{i j}=-\bar{u}_{i} u_{k} \partial \bar{U}_{k} / \partial x_{j}-\bar{u}_{j} u_{k} \partial \bar{U}_{k} / \partial x_{i}$ and $P_{i j}$ is the production tensor. The recommended values of the constants are $c_{2}=-0.44, c_{3}=0.46$, and $c_{4}=-0.23 .^{6}$ The term in $c_{5}$ has no effect for constant density flows but may be important

TABLE II. Coefficients $A^{(n)}$ of the tensors $T_{i j}^{(n)}$ for the FL model and definitions of scalar invariants $Q_{1}$ and $Q_{2}$.

\begin{tabular}{lc}
\hline \hline$A^{(1)}=-2.4 Q_{1}+0.8 Q_{2} c_{2}^{\prime}$ & $A^{(6)}=0.8-2 c_{2}^{\prime}$ \\
$A^{(2)}=0.8 Q_{1} c_{2}^{\prime}$ & $A^{(7)}=0.8+\frac{34}{15} c_{2}^{\prime}$ \\
$A^{(3)}=0.8+\frac{4}{3} b_{k k}^{2} c_{2}^{\prime}$ & $A^{(8)}=-1.6+3.2 c_{2}^{\prime}$ \\
$A^{(4)}=1.2+\left(0.4+2 b_{k k}^{2}\right) c_{2}^{\prime}$ & $A^{(9)}=-48 c_{2}-8 c_{2}^{\prime}$ \\
$A^{(5)}=\frac{26}{15}+16 b_{k k}^{2} c_{2}+\left(2 b_{k k}^{2}-\frac{14}{45}\right) c_{2}^{\prime}$ & $A^{(10)}=-4.8 c_{2}^{\prime}$ \\
$Q_{1}=b_{k l}^{2} S_{k l}-\frac{2}{3} b_{k l} S_{k l}-\frac{1}{3} S_{l l}$ & $Q_{2}=b_{k l} S_{k l}+\frac{1}{3} S_{l l}$ \\
\hline \hline
\end{tabular}

TABLE III. Generalized Langevin model coefficients for FL choosing $\beta_{1}=\gamma_{1}=\gamma_{4}=\xi_{1}=0$ and $\gamma_{5}$ to be a free parameter.

$$
\begin{aligned}
& \beta_{2}=\frac{11}{15}+4 b_{k k}^{2} c_{2}+\left(b_{k k}^{2}-\frac{8}{15}\right) c_{2}^{\prime}+\frac{1}{3} \gamma_{5} \\
& \beta_{3}=-\frac{2}{15}-4 b_{k k}^{2} c_{2}+\frac{8}{15} c_{2}^{\prime}-\frac{1}{3} \gamma_{5} \\
& \gamma_{2}=0.4+\frac{1}{15} c_{2}^{\prime} \\
& \gamma_{3}=-\frac{16}{15} c_{2}^{\prime} \\
& \gamma_{6}=-0.4+1.6 c_{2}^{\prime}-\gamma_{5} \\
& \xi_{2}=12 c_{2}+0.8 c_{2}^{\prime} \\
& \xi_{3}=-12 c_{2}-3.2 c_{2}^{\prime}
\end{aligned}
$$

in flows with large density variations. In general representation the non-zero coefficients of this model read as

$$
\begin{aligned}
& A^{(1)}=2 c_{5} S_{k k}, \quad A^{(3)}=-2 c_{2}+2 c_{4}, \\
& A^{(4)}=-3 c_{2}, \quad A^{(5)}=3 c_{2}+4 c_{3},
\end{aligned}
$$

which yields $A^{*}=3 c_{4}$. This implies that an equivalent GLM of JM exists only if $c_{4}=0$. Expansion in tensor polynomials shows that $c_{4}$ multiplies $T_{i j}^{(3)}$ which is a zero-th order term in the anisotropy tensor. The condition $c_{4}=0$ expresses that, in a GLM [Eq. (5)], the zero-th and first order tensors $T_{i j}^{(3)}$ and $T_{i j}^{(4)}$ are not independent. Implications for realizability are discussed below. Choosing $\gamma_{5}=0$ the non-zero GLM coefficients are given by

$$
\beta_{2}=c_{3}, \quad \beta_{3}=-\frac{3}{2} c_{2}-c_{3}
$$

Note that for variable density flows terms $\delta_{k l}$ in Eq. (4) multiply $\partial \bar{U}_{l} / \partial x_{l}$ which is non-zero.

We now have a closer look at some implications of three realizability constraints, which were used to derive the FL model, on the relationship between second-moment closure models and Langevin models. Because the Langevin model predicts the velocity of a realization of a fluid particle the model always predicts realizable states of turbulence as long as the model coefficients remain finite. Therefore a Langevin equivalent of a second-moment closure can exist only if this model is realizable.

The constraint that the model is redistributive $\left(a_{l i k i}=0\right)$ only or, in other words, the model may not affect the level of turbulent kinetic energy but may only redistribute the energy over the stress components, is always satisfied by a model which can be represented in the tensor polynomials $T_{i j}^{(n)}$. By construction the tensors $T_{i j}^{(n)}$ have zero trace and the model is redistributive. The GLM always satisfies this constraint because the condition that the modeled redistribution term has zero trace is used to determine the coefficients $\alpha$.

The constraint of normalization $\left(a_{l i k k}=\bar{u}_{i} u_{l} / k\right)$ is difficult to apply to a model in general representation because the relationship between the fourth-order tensor $a_{l i k j}$ in Eq. (1) and the second-order tensors $T_{i j}^{(n)}$ is not explicit. Concentrating on the rapid term, which is linear in mean velocity gradients, the relationship between the $a_{l i k j}$ in Eq. (1) and $H_{i j k l}$ in Eq. (3) is given by

$$
2 k\left(a_{l i k j}+a_{l j k i}\right)=H_{i m k l} \bar{u}_{j} u_{m}+H_{j m k l}{\overline{u_{i}}}_{m},
$$


in which terms in $\alpha$ which contain scalar invariants of $S_{i j}$ are represented in $H_{i j k l}$. There is no explicit relation between $a_{l i k j}$ and $H_{i j k l}$ and the direct implications of this constraint on the GLM coefficients are unknown.

The rapid pressure-strain term modeled by the GLM is given by the RHS of Eq. (10) multiplied by $\partial \bar{U}_{k} / \partial x_{l}$. It is easily seen that this model always satisfies realizability at the $2 \mathrm{D}$ turbulence limit ( $\phi_{\alpha \alpha 2}=0$ for $\bar{u}_{\alpha} u_{\alpha} \rightarrow 0$ ). Consequently, a representation of a conventional rapid pressure-strain model by a GLM exists only if this model is realizable at the 2D limit. Application of the constraint to an expression in general representation yields that $S_{\alpha \alpha}$ multiplies $A^{*}$ and realizability can be satisfied only if Eq. (6) is valid. The fact that this condition is necessary for the existence of a GLM representation of a second-moment closure has already been shown. In general JM does not satisfy realizability at the 2D turbulence limit for $c_{4} \neq 0$. Usually the $2 \mathrm{D}$ limit is approached near walls only where $S_{\alpha \alpha}$ is zero and a non-zero value of $A^{*}$ will not violate realizability. Jones and Musonge ${ }^{5}$ suggest to make $c_{4}$ a function of the anisotropy invariants such that it goes to zero at the $1 \mathrm{D}$ and $2 \mathrm{D}$ limits. The total redistribution term modeled by the GLM satisfies realizability in the sense that the $2 \mathrm{D}$ limit can never be reached. ${ }^{7}$ At the 2D limit realizability is not satisfied because of the assumption of local isotropy at the smallest scales, which is modeled by the isotropic Wiener process, which will always disturb the 2D state of turbulence. This property of the GLM becomes clear mathematically by the fact that, in general, the coefficients $\alpha$ become infinite for 2D turbulence. Dreeben and Pope $^{8}$ calculate near wall flows using a GLM in which they overcome this problem by modifying the constant $C_{0}$.

The GLM as defined by Haworth and Pope ${ }^{2}$ and which is extended here by adding quadratic terms in the anisotropy tensor, is able to represent the rapid pressure-strain correlation model of $\mathrm{Fu}$ and Launder. The Jones and Musonge model can be represented by a GLM only if the model pa- rameter $c_{4}$ equals zero. Because the GLM prediction of the rapid pressure-strain term always satisfies realizability at the two dimensional turbulence limit the corresponding secondmoment closure has to satisfy this constraint also. JM satisfies this constraint for $c_{4}=0$ only. The total redistribution term which accounts for both rapid pressure-strain and "return-to-isotropy" effects, as predicted by the GLM, cannot satisfy realizability at the 2D limit because of the isotropy of the Wiener process. However realizability is satisfied in the sense that the 2D limit cannot be reached in a finite time. The realizability constraint of redistribution is needed as an extra condition to uniquely specify the GLM coefficients $\alpha$. Most published second-moment closure models satisfy this constraint. The implications of the normalization constraint for the relationship between Langevin models and second-moment closures remains a topic for further research.

${ }^{a}$ Corresponding author. Section Heat Transfer, Department of Applied Physics, Delft University of Technology, Lorentzweg 1, 2628 CJ, Delft, the Netherlands. Telephone: +31-15-278 6125; fax: +31-15-278 1204; electronic mail: huib@duttwta.tn.tudelft.nl

b) Also at Shell Research and Technology Centre, Amsterdam, the Netherlands.

${ }^{1}$ S. B. Pope, "Lagrangian methods for turbulent flows," Annu. Rev. Fluid Mech. 26, 23 (1994).

${ }^{2}$ D. C. Haworth and S. B. Pope, "A generalized Langevin model for turbulent flows," Phys. Fluids 29, 387 (1986).

${ }^{3}$ S. B. Pope, "On the relationship between stochastic Lagrangian models of turbulence and second-moment closures," Phys. Fluids 6, 973 (1994).

${ }^{4} \mathrm{~S}$. Fu, Ph.D. thesis, University of Manchester Institute of Scientific Technology, 1988.

${ }^{5}$ W. P. Jones and P. Musonge, "Closure of the Reynolds stress and scalar flux equations," Phys. Fluids 31, 3589 (1988).

${ }^{6}$ W. P. Jones, in Turbulent Reacting Flows, edited by P. A. Libby and F. A. Williams (Academic Press, London, 1994).

${ }^{7}$ C. G. Speziale, R. Abid, and P. A. Durbin, "New results on the realizability of Reynolds stress turbulence closures," ICASE Report No. 93-76, 1993.

${ }^{8}$ T. D. Dreeben and S. B. Pope, "PDF and Reynolds stress modeling of near-wall turbulent flows," 10th Symposium on Turbulent Shear Flows, 1995, p. 2-1. 